\title{
Real and complex indices of vector fields on complete intersection curves with isolated singularity
}

\author{
Oliver Klehn
}

\begin{abstract}
If $(V, 0)$ is an isolated complete intersection singularity and $X$ a holomorphic vector field tangent to $V$, one can define an index of $X$, the so-called GSV index, which generalizes the Poincaré-Hopf index. We prove that the GSV index coincides with the dimension of a certain explicitly constructed vector space, if $X$ is deformable in a certain sense and $V$ is a curve. We also give a sufficient algebraic criterion for $X$ to be deformable in this way. If one considers the real analytic case one can also define an index of $X$ which is called the real GSV index. Under the condition that $X$ has the deformation property, we prove a signature formula for the index generalizing the Eisenbud-Levine Theorem.
\end{abstract}

\section{Introduction}

\subsection{Classical results}

Assume that the continuous map germ $g:\left(\mathbb{R}^{n}, 0\right) \rightarrow\left(\mathbb{R}^{n}, 0\right)$ defines an isolated zero. Then the map $g /\|g\|: S_{\delta}^{n-1} \rightarrow S^{n-1}$ of spheres around the origin has a degree, the so-called Poincaré-Hopf index $\operatorname{ind}_{\mathbb{R}^{n}, 0}(g)$ of $g$. If $g$ is analytic one has algebraic interpretations of this index, that we first want to describe. If $g:\left(\mathbb{C}^{n}, 0\right) \rightarrow\left(\mathbb{C}^{n}, 0\right)$ is holomorphic, let $Q_{g}$ be the algebra obtained by factoring $\mathscr{O}_{\mathbb{C}^{n}, 0}$ by the ideal generated by the components of $g$. One has the following theorem.

Theorem 1.1 [AGV85, GH78].

$$
\operatorname{ind}_{\mathbb{C}^{n}, 0}(g)=\operatorname{dim}_{\mathbb{C}} Q_{g}
$$

Here we have made the identification $\mathbb{C}^{n} \cong \mathbb{R}^{2 n}$ of course. Now let $\mathscr{E}_{\mathbb{R}^{n}, 0}$ be the ring of real analytic function germs on $\left(\mathbb{R}^{n}, 0\right)$ and further $g:\left(\mathbb{R}^{n}, 0\right) \rightarrow\left(\mathbb{R}^{n}, 0\right)$ be finite and real analytic, in the sense that $Q_{g}$ is finite dimensional as an $\mathbb{R}$-vector space and where $Q_{g}$ is the algebra obtained by factoring $\mathscr{E} \mathbb{R}^{n}, 0$ with the ideal generated by the components of $g$ in this case. If one denotes by $J_{g}$ the determinant of the Jacobian of $g$, one has the following famous theorem:

Theorem 1.2 (Eisenbud-Levine Theorem). Let $l: Q_{g} \rightarrow \mathbb{R}$ be a linear form with $l\left(J_{g}\right)>0$. Then

$$
\operatorname{ind}_{\mathbb{R}^{n}, 0}(g)=\text { signature }\langle,\rangle_{l} \text {. }
$$

Here $\langle,\rangle_{l}$ is the induced bilinear form defined by $\left\langle h_{1}, h_{2}\right\rangle_{l}:=l\left(h_{1} \cdot h_{2}\right)$.

\subsection{Generalization to complete intersections}

Now let $(V, 0):=\left(\left\{f_{1}=\cdots=f_{q}=0\right\}, 0\right) \subset\left(\mathbb{C}^{n}, 0\right)$ be an isolated singularity of a complete intersection (ICIS) and $X:=\sum_{i=1}^{n} X_{i} \partial / \partial z_{i}$ be the germ of a holomorphic vector field on $\left(\mathbb{C}^{n}, 0\right)$ tangent to $V$, say $X f=C f$ with an isolated zero on $V$. In this situation one can also define an

Received 28 January 2003, accepted in final form 30 January 2004, published online 10 February 2005.

2000 Mathematics Subject Classification 32S65 (primary), 14B05, $13 \mathrm{H} 10$ (secondary).

Keywords: index, vector field, local residue, signature, socle, isolated singularity.

This journal is (C) Foundation Compositio Mathematica 2005. 


\section{O. KLEHN}

index $\operatorname{ind}_{V, 0}(X)$, called the (complex) GSV index (see [ASV98, BG94, GSV91]); and it is the Poincaré-Hopf index when $V$ is smooth. The definition of the index is as follows:

Choose a sufficiently small sphere $S_{\delta}$ around the origin in $\mathbb{C}^{n}$ which intersects $V$ transversally and consider the link $K=V \cap S_{\delta}$ of $V$. The vectors $X, \nabla f_{1}, \ldots, \nabla f_{q}$ are linearly independent for all points of $K$ and we have a well defined map

$$
\left(X, \nabla f_{1}, \ldots, \nabla f_{q}\right): K \rightarrow W_{q+1}\left(\mathbb{C}^{n}\right),
$$

where $W_{q+1}\left(\mathbb{C}^{n}\right)$ denotes the manifold of $(q+1)$-frames in $\mathbb{C}^{n}$ and we consider the complex gradients of course. We have

$$
H_{2 n-2 q-1}(K) \cong \mathbb{Z}, \quad H_{2 n-2 q-1}\left(W_{q+1}\left(\mathbb{C}^{n}\right)\right) \cong \mathbb{Z},
$$

and therefore the map has a degree. We let $K$ be oriented as the boundary of the complex manifold $V \backslash\{0\}$ here. The index $\operatorname{ind}_{V, 0}(X)$ of $X$ is defined to be the degree of this map. (If $V$ is a curve, $K$ can have more components; we will then sum over the degrees of the components.)

We now want to formulate our main theorems. We need a definition first.

Definition 1.3. We have that $X$ is called a good vector field (with respect to $V$ ), if there is a holomorphic deformation $X_{t}$ of $X$, so that for all $t \in \mathbb{C}^{q}$ sufficiently close to zero $X_{t}$ is tangent to the $t$-fibre $V_{t}$ of $f$. Then $X_{t}$ is called a good deformation of $X$.

We will prove a sufficient criterion for a vector field to be good, which states that $X$ is good whenever all coefficients of the matrix $C$ are contained in the ideal generated by the maximal minors of the Jacobian of $f$ in $\mathscr{O}_{\mathbb{C}^{n}, 0}$. It follows from the definition of the index that it equals the sum of the indices of a good deformation on a smooth fibre.

After a linear generic change of coordinates one can assume that $\left(f_{1}, \ldots, f_{q}, X_{1}, \ldots X_{n-q}\right)$ is a regular $\mathscr{O}_{\mathbb{C}^{n}, 0}$-sequence (see [LSS95]), and we always assume the coordinates to be chosen in this way in this paper. Let $\mathscr{B}_{0}:=\mathscr{O}_{\mathbb{C}^{n}, 0} /\left(f_{1}, \ldots, f_{q}, X_{1}, \ldots, X_{n-q}\right)$. Due to the chosen coordinates $\mathscr{B}_{0}$ is finite dimensional as a complex vector space. We also set $\mathscr{C}_{0}:=\mathscr{B}_{0} / \operatorname{ann}_{\mathscr{B}_{0}}(D F)$, where

$$
D F:=\operatorname{det}\left(\frac{\partial\left(f_{1}, \ldots, f_{q}\right)}{\partial\left(z_{n-q+1}, \ldots, z_{n}\right)}\right) .
$$

We prove (in $\S 4.3$ ) an index formula for vector fields in the case $q=n-1$ :

Theorem 1.4. Let $X$ be a good vector field and $V$ a curve. Then

$$
\operatorname{ind}_{V, 0}(X)=\operatorname{dim}_{\mathbb{C}} \mathscr{C}_{0} .
$$

Now let

$$
\left(V^{\mathbb{R}}, 0\right):=\left(\left\{f_{1}^{\mathbb{R}}=\cdots=f_{q}^{\mathbb{R}}=0\right\}, 0\right) \subset\left(\mathbb{R}^{n}, 0\right),
$$

defined by real analytic function germs. If $f$ denotes the complexification of $f^{\mathbb{R}}$ we assume that $f$ defines an ICIS of dimension $n-q$. Furthermore let the real analytic vector field $X^{\mathbb{R}}$ be tangent to $\left(V_{\mathbb{R}}, 0\right)$ with an algebraic isolated zero on $\left(V^{\mathbb{R}}, 0\right)$. One defines the real GSV index of $X^{\mathbb{R}}$ similarly to the complex index (see [ASV98]), and denotes this index by $\operatorname{ind}_{V^{\mathbb{R}, 0},}\left(X^{\mathbb{R}}\right)$ if $n-q$ is odd and by $\operatorname{ind}_{V^{\mathbb{R}, 0}}^{2}\left(X^{\mathbb{R}}\right)$ if $n-q$ is even. For topological reasons one can only define a $(\bmod 2)$-index if $n-q$ is even. The definition of $X^{\mathbb{R}}$ to be good is as in the complex case using real analytic deformations.

For the case $q=n-1$ we prove (in $\S 5.1$ ) the following formula generalizing the Eisenbud-Levine Theorem:

TheOREm 1.5. Let $V^{\mathbb{R}}$ be a curve, $X^{\mathbb{R}}$ a good vector field and $l: \mathscr{C}_{0}^{\mathbb{R}} \rightarrow \mathbb{R}$ a linear form with $l\left(c_{1}\right)>0$. Then

$$
\operatorname{ind}_{V^{\mathbb{R}}, 0}\left(X^{\mathbb{R}}\right)=\text { signature }\langle,\rangle_{l}
$$




\section{REAL AND COMPLEX INDICES OF VECTOR FIELDS ON CURVES}

Here $\mathscr{C}_{0}^{\mathbb{R}}$ is defined as in the complex case using $\mathscr{E}_{\mathbb{R}^{n}, 0}$ instead of $\mathscr{O}_{\mathbb{C}^{n}, 0}, c_{1}$ is the coefficient of $t$ in the formal power series expansion of $\operatorname{det}\left(\nVdash+t D X^{\mathbb{R}}\right) / \operatorname{det}(\nVdash+t C)$, where $D X^{\mathbb{R}}$ is the Jacobian of $X^{\mathbb{R}}$, and $\langle,\rangle_{l}$ is the induced bilinear form defined as in the classical case. Finally, $C$ is defined by the tangency condition $X^{\mathbb{R}} f^{\mathbb{R}}=C f^{\mathbb{R}}$.

\section{Residues of holomorphic vector fields}

To prove our main theorems we need a few results on residues of holomorphic vector fields that we want to collect in this section.

Let $g:\left(\mathbb{C}^{n}, 0\right) \rightarrow\left(\mathbb{C}^{n}, 0\right)$ be a holomorphic map germ with isolated zero. Then the residue $\operatorname{res}_{\mathbb{C}^{n}, 0}^{g}(h)$ of any $h \in \mathscr{O}_{\mathbb{C}^{n}, 0}$ with respect to $g$ is defined as

$$
\operatorname{res}_{\mathbb{C}^{n}, 0}^{g}(h):=\frac{1}{(2 \pi i)^{n}} \int_{\Gamma} \frac{h d z_{1} \wedge \cdots \wedge d z_{n}}{g_{1} \cdot \ldots \cdot g_{n}},
$$

where $\Gamma$ is the real $n$-cycle $\Gamma:=\left\{\left|g_{i}\right|=\epsilon_{i}, i=1, \ldots, n\right\}$ for $\epsilon_{i} \in \mathbb{R}_{>0}$ small enough with orientation given by $d\left(\arg g_{1}\right) \wedge \cdots \wedge d\left(\arg g_{n}\right) \geqslant 0$. Sometimes we denote this residue also by

$$
\operatorname{res}_{\mathbb{C}^{n}, 0}\left[\begin{array}{c}
h \\
g_{1} \cdots g_{n}
\end{array}\right] \text {. }
$$

If we denote by $J_{g}$ the Jacobian determinant of $g$, we have the following classical result:

TheOrem 2.1 [AGV85, GH78].

i) The residue $\operatorname{res}_{\mathbb{C}^{n}, 0}^{g}: Q_{g} \rightarrow \mathbb{C}$ defines a linear form.

ii) The induced bilinear form $\langle,\rangle_{\operatorname{res}_{\mathbb{C}^{n}, 0}^{g}}$ is non-degenerate.

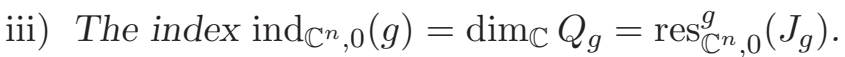

If we consider linear forms $l: Q \rightarrow \mathbb{F}$ on commutative $\mathbb{F}$-algebras for an arbitrary field in this paper, the induced bilinear form $\langle,\rangle_{l}$ on $Q$ is always the bilinear form defined by $\left\langle h_{1}, h_{2}\right\rangle_{l}:=l\left(h_{1} \cdot h_{2}\right)$. The second statement in the theorem is usually called 'local (Grothendieck) duality' and it states that $Q_{g}$ is a Gorenstein algebra. This means that the annihilator of the maximal ideal, the socle, of $Q_{g}$ is one-dimensional and it is well known that it is generated by the class of $J_{g}$. One immediately concludes that for any linear form $l: Q_{g} \rightarrow \mathbb{C}$ with $l\left(J_{g}\right) \neq 0$ the induced pairing $\langle,\rangle_{l}$ is nondegenerate.

As in the second part of the Introduction, let $(V, 0):=\left(\left\{f_{1}=\cdots=f_{q}=0\right\}, 0\right) \subset\left(\mathbb{C}^{n}, 0\right)$ be an isolated singularity of a complete intersection (ICIS) and $X:=\sum_{i=1}^{n} X_{i} \partial / \partial z_{i}$ be the germ of a holomorphic vector field on $\left(\mathbb{C}^{n}, 0\right)$ tangent to $V$, say $X f=C f$ with an isolated zero on $V$. Further let

$$
\Sigma:=\left\{f_{1}=\cdots=f_{q}=0,\left|X_{1}\right|=\epsilon_{1}, \ldots,\left|X_{n-q}\right|=\epsilon_{n-q}\right\}
$$

be a small real $(n-q)$-cycle with orientation determined by

$$
d\left(\arg X_{1}\right) \wedge \cdots \wedge d\left(\arg X_{n-q}\right) \geqslant 0 .
$$

Then we define the relative residue of any $h \in \mathscr{O}_{\mathbb{C}^{n}, 0}$ with respect to $X$ to be

$$
\operatorname{res}_{V, 0}^{X}(h):=\frac{1}{(2 \pi i)^{n-q}} \int_{\Sigma} \frac{h d z_{1} \wedge \cdots \wedge d z_{n-q}}{X_{1} \cdot \cdots \cdot X_{n-q}} .
$$

The absolute residue of $h$ with respect to $X$ is defined as

$$
\operatorname{res}_{\mathbb{C}^{n}, 0}^{X}(h):=\operatorname{res}_{\mathbb{C}^{n}, 0}\left[\begin{array}{c}
h \\
X_{1} \cdots X_{n-q} f_{1} \cdots f_{q}
\end{array}\right] \text {. }
$$




\section{O. KLEHN}

Now let $c_{n-q}$ be the coefficient of $t^{n-q}$ in the formal power series expansion of

$$
\operatorname{det}(\nVdash+t D X) / \operatorname{det}(\nVdash+t C),
$$

where $D X$ is the Jacobian of $X$. We have the following theorem proven in [LSS95].

THEOREM 2.2 [LSS95].

$$
\operatorname{ind}_{V, 0}(X)=\operatorname{res}_{V, 0}^{X}\left(c_{n-q}\right) .
$$

The author has proven in [Kle02] that one always has $\operatorname{res}_{V, 0}^{X}(h)=\operatorname{res}_{\mathbb{C}^{n}, 0}^{X}(h D F)$, where we have set

$$
D F:=\operatorname{det}\left(\frac{\partial\left(f_{1}, \ldots, f_{q}\right)}{\partial\left(z_{n-q+1}, \ldots, z_{n}\right)}\right)
$$

This means that we also have $\operatorname{ind}_{V, 0}(X)=\operatorname{res}_{\mathbb{C}^{n}, 0}^{X}\left(c_{n-q} D F\right)$, which is one of the main tools in the proof of our main theorems.

\section{Vector fields tangent to smooth varieties}

To prove our main theorems we first prove them for the smooth situation, which is done in this section, and use good deformations to generalize to the singular case. We also look at the socle of $\mathscr{C}_{0}$. We use the notation as introduced in the first and second sections.

\subsection{The complex situation}

Let $1 \leqslant i_{1}<\cdots<i_{q} \leqslant n$ be fixed and $1 \leqslant j_{1}<\cdots<j_{n-q} \leqslant n$ be the complement of $i_{1}, \ldots, i_{q}$ in $\{1, \ldots, n\}$. Furthermore we set

$$
D I:=\operatorname{det}\left(\frac{\partial\left(f_{1}, \ldots, f_{q}\right)}{\partial\left(z_{i_{1}}, \ldots, z_{i_{q}}\right)}\right)
$$

and let $\sigma_{I}$ be the permutation defined by

$$
\sigma_{I}:=\left(\begin{array}{cccccc}
1 & \ldots & n-q & n-q+1 & \ldots & n \\
j_{1} & \ldots & j_{n-q} & i_{1} & \ldots & i_{q}
\end{array}\right),
$$

where $I$ is the multiindex $I:=\left(i_{1}, \ldots, i_{q}\right)$. We also set

$$
\mathscr{B}_{0}^{I}:=\frac{\mathscr{O}_{\mathbb{C}^{n}, 0}}{\left(f_{1}, \ldots, f_{q}, X_{j_{1}}, \ldots, X_{j_{n-q}}\right)}
$$

and if $D I(0) \neq 0$

$$
\gamma_{I}:=\frac{\operatorname{sign} \sigma_{I}}{D I} \operatorname{det}\left(\frac{\partial\left(X_{j_{1}}, \ldots, X_{j_{n-q}}, f_{1}, \ldots, f_{q}\right)}{\partial\left(z_{1}, \ldots, z_{n}\right)}\right) .
$$

Lemma 3.1. Let $D I(0) \neq 0$. Then

$$
\operatorname{ind}_{V, 0}(X)=\operatorname{dim}_{\mathbb{C}} \mathscr{B}_{0}^{I} .
$$

Proof. By the implicit mapping theorem it is not hard to show that $\left.X\right|_{V}$ corresponds to the vector field

$$
Y:=X_{j_{1}} \circ \psi \frac{\partial}{\partial y_{1}}+\cdots+X_{j_{n-q}} \circ \psi \frac{\partial}{\partial y_{n-q}}
$$

on $\left(\mathbb{C}^{n-q}, 0\right)$, where $\psi:\left(\mathbb{C}^{n-q}, 0\right) \rightarrow(V, 0)$ is a biholomorphic map as in the implicit mapping theorem. From

the claim follows.

$$
\frac{\mathscr{O}_{\mathbb{C}^{n-q}, 0}}{\left(X_{j_{1}} \circ \psi, \ldots, X_{j_{n-q}} \circ \psi\right)} \cong \mathscr{B}_{0}^{I}
$$




\section{REAL AND COMPLEX INDICES OF VECTOR FIELDS ON CURVES}

Lemma 3.2. Let $D I(0) \neq 0$. Then we have for any $h \in \mathscr{O}_{\mathbb{C}^{n}, 0}$

$$
\operatorname{res}_{\mathbb{C}^{n}, 0}^{X}(h D F)=\operatorname{sign} \sigma_{I} \operatorname{res}_{\mathbb{C}^{n}, 0}\left[\begin{array}{c}
h D I \\
X_{j_{1}} \ldots X_{j_{n-q}} f_{1} \ldots f_{q}
\end{array}\right] .
$$

Proof. By the transformation formula for residues [GH78], we have to show that there is a matrix $A$ with

$$
\left(X_{1}, \ldots, X_{n-q}, f_{1}, \ldots, f_{q}\right)^{\mathrm{t}} A\left(X_{j_{1}}, \ldots, X_{j_{n-q}}, f_{1}, \ldots, f_{q}\right)^{\mathrm{t}}
$$

and $\operatorname{det} A=\operatorname{sign} \sigma_{I} D F / D I$. From $X f=C f$ we get

$$
\left(\begin{array}{c}
X_{i_{1}} \\
\vdots \\
X_{i_{q}}
\end{array}\right)=C f-\left(\frac{\partial\left(f_{1}, \ldots, f_{q}\right)}{\partial\left(z_{i_{1}}, \ldots, z_{i_{q}}\right)}\right)^{-1} \frac{\partial\left(f_{1}, \ldots, f_{q}\right)}{\partial\left(z_{j_{1}}, \ldots, z_{j_{n-q}}\right)}\left(\begin{array}{c}
X_{j_{1}} \\
\vdots \\
X_{j_{n-q}}
\end{array}\right) .
$$

Now let $i_{1}, \ldots, i_{k} \in\{1, \ldots, n-q\}$ and $i_{k+1}, \ldots, i_{q} \in\{n-q+1, \ldots, n\}$. Then it follows that $j_{1}, \ldots, j_{n-q-k} \in\{1, \ldots, n-q\}$ and $j_{n-q-k+1}, \ldots, j_{n-q} \in\{n-q+1, \ldots, n\}$. For $k=0$ the claim follows immediately. With (1) we obtain a matrix $B$ with

$$
\left(X_{j_{1}}, \ldots, X_{j_{n-q+k}}, X_{i_{1}}, \ldots, X_{i_{k}}, f_{1}, \ldots, f_{q}\right)^{\mathrm{t}}=B\left(X_{j_{1}}, \ldots, X_{j_{n-q}}, f_{1}, \ldots, f_{q}\right)^{\mathrm{t}} .
$$

If $\sigma^{\prime} \in S_{n-q}$ is the permutation with

$$
\sigma^{\prime}=\left(\begin{array}{cccccc}
1 & \ldots & n-q+k & n-q+k+1 & \ldots & n-q \\
j_{1} & \ldots & j_{n-q-k} & i_{1} & \ldots & i_{k}
\end{array}\right),
$$

we have $\operatorname{det} A=\operatorname{sign} \sigma^{\prime} \operatorname{det} B$. Then $\operatorname{det} B$ is the determinant of the upper right $(k \times k)$ block of the matrix

$$
-\left(\frac{\partial\left(f_{1}, \ldots, f_{q}\right)}{\partial\left(z_{i_{1}}, \ldots, z_{i_{q}}\right)}\right)^{-1} \frac{\partial\left(f_{1}, \ldots, f_{q}\right)}{\partial\left(z_{j_{1}}, \ldots, z_{j_{n-q}}\right)} .
$$

If $d_{l, m}$ is the determinant of the matrix obtained by replacing the $l$ th column of

$$
\frac{\partial\left(f_{1}, \ldots, f_{q}\right)}{\partial\left(z_{i_{1}}, \ldots, z_{i_{q}}\right)}
$$

by the $m$ th column of

$$
\frac{\partial\left(f_{1}, \ldots, f_{q}\right)}{\partial\left(z_{j_{1}}, \ldots, z_{j_{n-q}}\right)}
$$

and if

$$
D:=\left(d_{l, m}\right)_{l=1, \ldots, k}^{m=n-q-k+1, \ldots, n-q},
$$

we have to show that

$$
\operatorname{det} D=(D I)^{k-1} \operatorname{det}\left(\frac{\partial\left(f_{1}, \ldots, f_{q}\right)}{\partial\left(z_{j_{n-q-k+1}}, \ldots, z_{j_{n-q}}, z_{i_{k+1}}, \ldots, z_{i_{q}}\right)}\right) .
$$

This can be done by induction over $k$ where $k=1$ is obvious. The conclusion is straightforward and we do not want to write it down here.

LEMMA 3.3. If $V$ is smooth, then

$$
\operatorname{ind}_{V, 0}(X)=\operatorname{dim}_{\mathbb{C}} \mathscr{C}_{0}
$$

Proof. Let $D I(0) \neq 0$. By Lemma 3.1 we have

$$
\operatorname{ind}_{V, 0}(X)=\operatorname{dim}_{\mathbb{C}} \mathscr{B}_{0}^{I} .
$$




\section{O. KLEHN}

If we map a class $[h] \in \mathscr{C}_{0}$ to the class $[h]^{\prime}$ of $h$ in $\mathscr{B}_{0}^{I}$ we obtain an isomorphism of $\mathbb{C}$-algebras, since by Lemma 3.2 and local duality the following holds:

$$
\begin{aligned}
{[h]=0 \quad \text { in } \mathscr{C}_{0} } & \Longleftrightarrow h D F \in \mathscr{O}_{\mathbb{C}^{n}, 0}\left(f_{1}, \ldots, f_{q}, X_{1}, \ldots, X_{n-q}\right) \\
& \Longleftrightarrow \operatorname{res}_{\mathbb{C}^{n}, 0}^{X}(g h D F)=0 \quad \text { for all } g \in \mathscr{O}_{\mathbb{C}^{n}, 0} \\
& \Longleftrightarrow \operatorname{res}_{\mathbb{C}^{n}, 0}\left[\begin{array}{c}
g h D I \\
X_{j_{1}} \ldots X_{j_{n-q}} f_{1} \ldots f_{q}
\end{array}\right]=0 \quad \text { for all } g \in \mathscr{O}_{\mathbb{C}^{n}, 0} \\
& \Longleftrightarrow h D I \in \mathscr{O}_{\mathbb{C}^{n}, 0}\left(X_{j_{1}}, \ldots X_{j_{n-q}}, f_{1}, \ldots, f_{q}\right) \\
& \Longleftrightarrow[h]^{\prime}=0 \quad \text { in } \mathscr{B}_{0}^{I}
\end{aligned}
$$

Lemma 3.4. Let $D I(0) \neq 0$. Then in $\mathscr{C}_{0}$ the equation $c_{n-q}=\gamma_{I}$ holds.

Proof. By the transformation formula for residues and Lemma 3.1 we have

$$
\operatorname{res}_{\mathbb{C}^{n}, 0}^{X}\left(D F \gamma_{I}\right)=\operatorname{ind}_{V, 0}(X)
$$

and further for $h \in \mathfrak{m}_{\mathscr{O}_{\mathbb{C}}, 0}$

$$
\operatorname{res}_{\mathbb{C}^{n}, 0}^{X}\left(D F \gamma_{I} h\right)=\operatorname{res}_{\mathbb{C}^{n}, 0}\left[\begin{array}{c}
h \operatorname{det}\left(\frac{\partial\left(X_{j_{1}}, \ldots, X_{j_{n-q}}, f_{1}, \ldots, f_{q}\right)}{\partial\left(z_{1}, \ldots, z_{n}\right)}\right) \\
X_{j_{1}} \ldots X_{j_{n-q}} f_{1} \ldots f_{q}
\end{array}\right]=0
$$

and therefore $D F \gamma_{I}$ generates the one-dimensional socle of $\mathscr{B}_{0}$. By the remarks of the Introduction we have

$$
\operatorname{res}_{\mathbb{C}^{n}, 0}^{X}\left(D F c_{n-q}\right)=\operatorname{ind}_{V, 0}(X),
$$

and therefore $D F c_{n-q} \neq 0$ in $\mathscr{B}_{0}$. Since $V$ is smooth there is a small deformation $X_{t}$ of $X$ tangent to $V$, so that $X_{t}$ has only simple zeros $p_{i}$ for sufficiently small $t$ on $V$ in a small neighbourhood of the origin. We can also assume that for these zeros $D I\left(p_{i}\right) \neq 0$ holds. For $h \in \mathfrak{m}_{\mathscr{O}_{\mathbb{C}^{n}, 0}}$ we have

$$
\begin{aligned}
\operatorname{res}_{\mathbb{C}^{n}, 0}^{X}\left(D F c_{n-q} h\right) & =\operatorname{res}_{\mathbb{C}^{n}, 0}\left[\begin{array}{c}
\operatorname{sign} \sigma_{I} D I c_{n-q} h \\
X_{j_{1}} \cdots X_{j_{n-q}} f_{1} \cdots f_{q}
\end{array}\right] \\
& =\lim _{t \rightarrow 0} \sum_{i} h\left(p_{i}\right) \operatorname{ind}_{V, p_{i}}\left(X_{t}\right) \\
& =0 .
\end{aligned}
$$

This follows from the continuous principle for residues, from the fact that the algebras

$$
\frac{\mathscr{O}_{\mathbb{C}^{n}, p_{i}}}{\left(X_{t, j_{1}}, \ldots, X_{t, j_{n-q}}, f_{1} \ldots, f_{q}\right)}
$$

are one-dimensional and by the transformation formula that $\operatorname{sign} \sigma_{I} D I c_{n-q}(t)$ is a unit in these algebras. Therefore $c_{n-q} D F$ generates the one-dimensional socle of $\mathscr{B}_{0}$ too and since we have

$$
\operatorname{res}_{\mathbb{C}^{n}, 0}^{X}\left(D F c_{n-q}\right)=\operatorname{res}_{\mathbb{C}^{n}, 0}^{X}\left(D F \gamma_{I}\right)
$$

it follows that $D F\left(c_{n-q}-\gamma_{I}\right)=0$ in $\mathscr{B}_{0}$ and therefore $c_{n-q}=\gamma_{I}$ in $\mathscr{C}_{0}$.

\subsection{The real analytic situation}

Lemma 3.5. Let $\left(V^{\mathbb{R}}, 0\right)$ be smooth and $l: \mathscr{C}_{0}^{\mathbb{R}} \rightarrow \mathbb{R}$ a linear form with $l\left(c_{n-q}\right)>0$. Then we have $\operatorname{ind}_{V^{\mathbb{R}}, 0}\left(X^{\mathbb{R}}\right)=$ signature $\langle,\rangle_{l}$.

Proof. Let $D I(0) \neq 0$. With the implicit mapping theorem it is not hard to show that the vector field $\left.X^{\mathbb{R}}\right|_{V^{\mathbb{R}}}$ corresponds to

$$
Y:=X_{j_{1}}^{\mathbb{R}} \circ \psi \frac{\partial}{\partial y_{1}}+\cdots+X_{j_{n-q}}^{\mathbb{R}} \circ \psi \frac{\partial}{\partial y_{n-q}},
$$


where $\psi:\left(\mathbb{R}^{n-q}, 0\right) \rightarrow\left(V^{\mathbb{R}}, 0\right)$ is a diffeomorphism with $\psi_{j_{k}}(y)=y_{j_{k}}$ for $k=1, \ldots, n-q$. By the chain rule one has

$$
\frac{\partial\left(\psi_{i_{1}}, \ldots, \psi_{i_{q}}\right)}{\partial\left(y_{1}, \ldots, y_{n-q}\right)}-\left(\frac{\partial\left(f_{1}^{\mathbb{R}}, \ldots, f_{q}^{\mathbb{R}}\right)}{\partial\left(x_{i_{1}}, \ldots, x_{i_{q}}\right)} \circ \psi\right)^{-1} \frac{\partial\left(f_{1}^{\mathbb{R}}, \ldots, f_{q}^{\mathbb{R}}\right)}{\partial\left(x_{j_{1}}, \ldots, x_{j_{n-q}}\right)} \circ \psi
$$

Applying the chain rule again we get

$$
\begin{aligned}
\frac{\partial\left(Y_{1}, \ldots, Y_{n-q}\right)}{\partial\left(y_{1}, \ldots, y_{n-q}\right)} \frac{\partial\left(X_{j_{1}}^{\mathbb{R}}, \ldots, X_{j_{n-q}}^{\mathbb{R}}\right)}{\partial\left(x_{j_{1}}, \ldots, x_{j_{n-q}}\right)} \circ \psi & \\
& \quad-\frac{\partial\left(X_{j_{1}}^{\mathbb{R}}, \ldots, X_{j_{n-q}}^{\mathbb{R}}\right)}{\partial\left(x_{i_{1}}, \ldots, x_{i_{q}}\right)} \circ \psi\left(\frac{\partial\left(f_{1}^{\mathbb{R}}, \ldots, f_{q}^{\mathbb{R}}\right)}{\partial\left(x_{i_{1}}, \ldots, x_{i_{q}}\right)} \circ \psi\right)^{-1} \frac{\partial\left(f_{1}^{\mathbb{R}}, \ldots, f_{q}^{\mathbb{R}}\right)}{\partial\left(x_{j_{1}}, \ldots, x_{j_{n-q}}\right)} \circ \psi .
\end{aligned}
$$

A well known lemma from linear algebra states

$$
\operatorname{det}\left(\begin{array}{cc}
A & B \\
C & D
\end{array}\right)=\operatorname{det} A \cdot \operatorname{det}\left(D-C A^{-1} B\right),
$$

where $A$ and $D$ are square and $A$ is invertible. The application of this lemma shows that the determinant of the Jacobian of $Y$ is given by $\gamma_{I} \circ \psi$. By the Eisenbud-Levine Theorem it follows that for any linear form

$$
\varphi: \frac{\mathscr{E}_{\mathbb{R}^{n-q}, 0}}{\left(Y_{1}, \ldots, Y_{n-q}\right)} \rightarrow \mathbb{R}
$$

with $\varphi\left(\gamma_{I} \circ \psi\right)>0$ the statement $\operatorname{ind}_{V^{\mathbb{R}, 0}}(X)=$ signature $\langle,\rangle_{\varphi}$ holds. The isomorphism of algebras given by $\psi$ shows that we have for any linear form $\Phi: \mathscr{B}_{0}^{I \mathbb{R}} \rightarrow \mathbb{R}$ with $\Phi\left(\gamma_{I}\right)>0$ the formula $\operatorname{ind}_{V^{\mathbb{R}, 0}}(X)=$ signature $\langle,\rangle_{\Phi}$ and this is also true in $\mathscr{C}_{0}^{\mathbb{R}}$, because the isomorphism of algebras in Lemma 3.3 also gives an isomorphism of the corresponding real algebras. On the other hand one has in $\mathscr{C}_{0}$ the equation $c_{n-q}=\gamma_{I}$ and this equation also holds in $\mathscr{C}_{0}^{\mathbb{R}}$. Therefore the statement follows.

\section{An algebraic formula for the complex index}

\subsection{Good vector fields}

First we want to prove a sufficient criterion for good vector fields.

Proposition 4.1 (Sufficient criterion for good vector fields). Let all coefficients of the matrix $C$ be contained in the ideal generated by the maximal minors of the Jacobian of $f$ in $\mathscr{O}_{\mathbb{C}^{n}, 0}$. Then $X$ is a good vector field.

Proof. We prove a bit more: there is a deformation $X_{t}$ of $X$ such that $X_{t}(f-t)=C(f-t)$ holds. For $\left(i_{1}, \ldots, i_{q}\right) \in\{1, \ldots, n\}^{q}$ and $\left(j_{1}, \ldots, j_{q-1}\right) \in\{1, \ldots, n\}^{q-1}$ define

$$
f_{i_{1}, \ldots, i_{q}}:=\operatorname{det}\left(\frac{\partial\left(f_{1}, \ldots, f_{q}\right)}{\partial\left(z_{i_{1}}, \ldots, z_{i_{q}}\right)}\right) \quad \text { and } \quad f_{j_{1}, \ldots, j_{q-1}}^{k}:=\operatorname{det}\left(\frac{\partial\left(f_{1}, \ldots, \hat{f}_{k}, \ldots, f_{q}\right)}{\partial\left(z_{j_{1}}, \ldots, z_{j_{q-1}}\right)}\right) \text {. }
$$

If $C=\left(c_{l, m}\right)$ let

$$
c_{l, m}=\sum_{\left(i_{1}, \ldots, i_{q}\right) \in\{1, \ldots, n\}^{q}} c_{i_{1}, \ldots, i_{q}}^{l, m} f_{i_{1}, \ldots, i_{q}} .
$$

For $k=1, \ldots, q-1$, we set

$$
\delta_{j_{1}, \ldots, j_{q-1}}^{i, j}:=\delta_{j_{1}, \ldots, j_{k-1}, i, j_{k+1}, \ldots, j_{q-1}}^{j_{k}, j}: \delta_{i, j_{1}, \ldots, j_{q-1}}^{j}
$$




\section{O. KLEHN}

and further

$$
\delta_{i_{1}, \ldots, i_{q}}^{l}:=-\sum_{m=1}^{q} \sum_{\left(i_{1}, \ldots, i_{q}\right) \in\{1, \ldots, n\}^{q}} c_{i_{1}, \ldots, i_{q}}^{l, m} t_{m} .
$$

We define the deformation by

$$
X_{t, i}:=X_{i}+\sum_{j=1}^{q} \sum_{\left(j_{1}, \ldots, j_{q-1}\right) \in\{1, \ldots, n\}^{q-1}} \delta_{j_{1}, \ldots, j_{q-1}}^{i, j}(-1)^{j+1} f_{j_{1}, \ldots, j_{q-1}}^{j} .
$$

Then we have

$$
\sum_{i=1}^{n} \frac{\partial f_{l}}{\partial z_{i}} X_{t, i} \sum_{m=1}^{n} c_{l, m} f_{m}+\sum_{\left(i_{1}, \ldots, i_{q}\right) \in\{1, \ldots, n\}^{q}} \delta_{i_{1}, \ldots, i_{q}}^{l} f_{i_{1}, \ldots i_{q}}=\sum_{m=1}^{n} c_{l, m}\left(f_{m}-t_{m}\right) .
$$

In the case of a hypersurface this means that, if $c$ is contained in the ideal generated by the partials of $f$, the vector field is good. If

$$
c=\alpha_{1} \frac{\partial f}{\partial z_{1}}+\cdots+\alpha_{n} \frac{\partial f}{\partial z_{n}}
$$

the deformation is simply defined as $X_{t, i}:=X_{i}-t \alpha_{i}$. We have

$$
X_{t}(f-t)=c f-\sum_{i=1}^{n} t \alpha_{i} \frac{\partial f}{\partial z_{i}}=c(f-t) .
$$

\subsection{The socle of $\mathscr{C}_{0}$}

Lemma 4.2. The residue $\operatorname{res}_{V, 0}^{X}(\cdot)$ defines a linear form on $\mathscr{C}_{0}$ such that

$$
\operatorname{res}_{V, 0}^{X}(h g)=0 \text { for all } h \in \mathscr{O}_{\mathbb{C}^{n}, 0} \Rightarrow g=0 \text { in } \mathscr{C}_{0}
$$

holds.

Proof. We have $\operatorname{res}_{V, 0}^{X}(h)=\operatorname{res}_{\mathbb{C}^{n}, 0}^{X}(h D F)$ and this means that the residue $\operatorname{res}_{V, 0}^{X}(\cdot)$ vanishes on $\operatorname{ann}_{\mathscr{B}_{0}}(D F)$. Furthermore $\operatorname{res}_{V, 0}^{X}(h g)=0$ for all $h \in \mathscr{O}_{\mathbb{C}^{n}, 0}$ implies $\operatorname{res}_{\mathbb{C}^{n}, 0}^{X}(h g D F)=0$ for all $h \in$ $\mathscr{O}_{\mathbb{C}^{n}, 0}$. Now local duality gives

$$
g D F \in \mathscr{O}_{\mathbb{C}^{n}, 0}\left(X_{1}, \ldots, X_{n-q}, f_{1}, \ldots, f_{q}\right)
$$

and therefore $g \in \operatorname{ann}_{\mathscr{B}_{0}}(D F)$.

Proposition 4.3. The socle of $\mathscr{C}_{0}$ is generated by the class of $c_{n-q}$.

Proof. Lemma 4.2 states that $\operatorname{res}_{V, 0}^{X}(\cdot)$ induces a non-degenerate bilinear form on $\mathscr{C}_{0}$, which means that $\mathscr{C}_{0}$ has a one-dimensional socle if $\mathscr{C}_{0}$ is not trivial. On the other hand for any $h \in \mathfrak{m}_{\mathscr{C}^{n}, 0}$ we have

$$
\operatorname{res}_{V, 0}^{X}\left(h c_{n-q}\right)=\lim _{t \rightarrow 0} \sum_{i} h\left(p_{i}\right) \operatorname{ind}_{V_{t}, p_{i}}\left(X_{t}\right)=0,
$$

which means that $c_{n-q}$ generates the socle.

\subsection{Proof of Theorem 1.4}

For a good vector field, $\operatorname{ind}_{V, 0}(X)$ is the sum of Poincaré-Hopf indices of $X_{t}$ on a smooth fibre $V_{t}$ of $f$, where one sums over all zeros of $X_{t}$ which tend to zero. This follows directly from the definition of the index.

If $q=n-1$ we denote by $m_{i}$ the minor of the Jacobian matrix of $f$ obtained by cancelling the $i$ th column. We have $D F=m_{1}$ of course. Recall that we always assume the coordinates to be 


\section{REAL AND COMPLEX INDICES OF VECTOR FIELDS ON CURVES}

chosen so that $\left(f_{1}, \ldots, f_{n-1}, X_{1}\right)$ is a regular $\mathscr{O}_{\mathbb{C}^{n}, 0}$-sequence. For simplicity we also may assume that $m_{i}(0)=0$ for $i=1, \ldots, n$.

Lemma 4.4. Let $q=n-1$. Then the following hold:

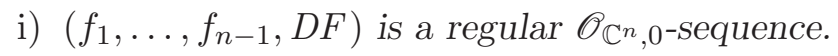

ii) The dimension of $\mathscr{C}_{0}$ does not depend on the choice of coordinates, provided that the $\mathscr{O}_{\mathbb{C}^{n}, 0}$-sequence $\left(f_{1}, \ldots, f_{n-1}, X_{1}\right)$ is regular in each system of coordinates.

iii) After linear changes of coordinates in $\mathbb{C}^{n}$ and $\mathbb{C}^{n-1}$ one can assume that $\left(f_{1}, \ldots, f_{n-2}, D F, m_{2}\right)$ is a regular $\mathscr{O}_{\mathbb{C}^{n}, 0}$-Sequence.

Proof. i) All computations are done in the ring $\mathscr{O}_{V, 0}=\mathscr{O}_{\mathbb{C}^{n}, 0} /\left(f_{1}, \ldots, f_{n-1}\right)$. We have to show that $D F$ is not a zero divisor in this ring. Applying Cramer's rule to the equation $X f=C f$ we obtain the equations

$$
(-1)^{i} m_{i} X_{1}=-D F X_{i}
$$

for $i=1, \ldots, n$. Now let $g D F=0$ in $\mathscr{O}_{V, 0}$. Multiplication with $X_{i}$, Equation (2) and using that $X_{1}$ is not a zero divisor in $\mathscr{O}_{V, 0}$ give $g m_{i}=0$ for all $i=1, \ldots, n$. The ring obtained by dividing $\mathscr{O}_{V, 0}$ by all $m_{i}$ is artinian and therefore there must be complex numbers $\alpha_{1}, \ldots, \alpha_{n}$ so that $h:=\alpha_{1} m_{1}+\cdots+\alpha_{n} m_{n}$ is not a zero divisor in $\mathscr{O}_{V, 0}$. On the other hand we have $g h=0$ in $\mathscr{O}_{V, 0}$ and therefore $g=0$ in $\mathscr{O}_{V, 0}$.

ii) Let $\phi:\left(\mathbb{C}^{n}, 0\right) \rightarrow\left(\mathbb{C}^{n}, 0\right), \phi(y)=z$, be biholomorphic and $\psi:=\phi^{-1}$. We denote by $Y$ the vector field computed in $y$-coordinates and by $D F^{y}$ the minor computed in $y$-coordinates. Standard computations give

$$
\left(\begin{array}{c}
Y_{1} \circ \psi \\
\vdots \\
Y_{n} \circ \psi
\end{array}\right)=\frac{\partial\left(\psi_{1}, \ldots, \psi_{n}\right)}{\partial\left(z_{1}, \ldots, z_{n}\right)}\left(\begin{array}{c}
X_{1} \\
\vdots \\
X_{n}
\end{array}\right)
$$

and

$$
D F^{y} \circ \psi=\sum_{j=1}^{n}(-1)^{j+1}(\operatorname{det} D \phi) \circ \psi \frac{\partial \psi_{1}}{\partial z_{j}} m_{j} .
$$

Set $\mathscr{B}_{0}^{\prime}:=\mathscr{O}_{\mathbb{C}^{n}, 0} /\left(f_{1}, \ldots, f_{n-1}, Y_{1} \circ \psi\right)$ and $\mathscr{C}_{0}^{\prime}:=\mathscr{B}_{0}^{\prime} / \operatorname{ann}_{\mathscr{B}_{0}^{\prime}}\left(D F^{y} \circ \psi\right)$. We construct an epimorphism $\varphi: \mathscr{C}_{0} \rightarrow \mathscr{C}_{0}^{\prime}$. For any $g \in \mathscr{O}_{\mathbb{C}^{n}, 0}$ we also denote by $g$ the classes of $g$ in these algebras and define $\varphi(g):=g$. Again all computations are done in the ring $\mathscr{O}_{V, 0}$. We want to show that $\varphi$ is well defined. Let $g D F=\alpha X_{1}$. Multiplying with $m_{i}$, using Equation (2) and the fact that $D F$ is not a zero divisor in $\mathscr{O}_{V, 0}$, we obtain $g m_{i}=(-1)^{i+1} \alpha X_{i}$ for $i=1, \ldots, n$. Then for each $i$ we get

$$
\begin{aligned}
g D F^{y} \circ \psi & =(\operatorname{det} D \phi) \circ \psi \sum_{i=1}^{n}(-1)^{i+1} \frac{\partial \psi_{1}}{\partial z_{i}} g m_{i} \\
& =(\operatorname{det} D \phi) \circ \psi \sum_{i=1}^{n} \alpha X_{i} \frac{\partial \psi_{1}}{\partial z_{i}} \\
& =\alpha(\operatorname{det} D \phi) \circ \psi Y_{1} \circ \psi .
\end{aligned}
$$

This shows that $\varphi$ is well defined. The surjectivity is obvious and the other direction analogous. Therefore $\operatorname{dim}_{\mathbb{C}} \mathscr{C}_{0}$ does not depend on the choice of coordinates in $\mathbb{C}^{n}$. If one considers changes of coordinates in the image space $\mathbb{C}^{n-1}$, the invariance of $\operatorname{dim}_{\mathbb{C}} \mathscr{C}_{0}$ is obvious.

iii) After a general linear change of coordinates in $\mathbb{C}^{n-1}$, see [Loo84], one can assume that $\left(f_{1}, \ldots, f_{n-2}\right)$ defines an ICIS and the 1 -form $d f_{n-1}$ has an isolated zero on this ICIS. Now Lemma 3.4 


\section{O. KLEHN}

in [Kle02] shows that, after a linear change of coordinates in $\mathbb{C}^{n},\left(f_{1}, \ldots, f_{n-2}, D F, m_{2}\right)$ is a regular $\mathscr{O}_{\mathbb{C}^{n}, 0 \text {-sequence. }}$

Proof of Theorem 1.4. Let $q=n-1$ and consider good deformations $X_{t}$ of $X$. For small neighbourhoods $U$ (respectively $T$ ) of the origins in $\mathbb{C}^{n}$ (respectively $\mathbb{C}^{n-1}$ ) consider $Z \subset U \times T$ defined by

$$
Z:=\left\{f_{t, 1}=\cdots=f_{t, n-1}=X_{t, 1}=0\right\}
$$

where we have set $f_{t, i}:=f_{i}-t_{i}$. Let $\pi: Z \rightarrow T$ be the finite projection. We define

$$
\mathscr{B}_{t, p}:=\frac{\mathscr{O}_{\mathbb{C}^{n}, p}}{\left(f_{t, 1}, \ldots, f_{t, n-1}, X_{t, 1}\right)}, \quad \mathscr{B}_{t}:=\bigoplus_{p \in \pi^{-1}(t)} \mathscr{B}_{t, p}, \quad \mathscr{B}:=\bigcup_{t \in T} \mathscr{B}_{t} .
$$

$\mathscr{B}$ has the natural structure of a holomorphic vector bundle over $T$ which is induced by the locally free sheaf $\pi_{*} \mathscr{O}_{Z}$. Similarly we define

$$
\mathscr{C}_{t, p}:=\frac{\mathscr{B}_{t, p}}{\operatorname{ann}_{\mathscr{B}_{t, p}}(D F)}, \quad \mathscr{C}_{t}:=\bigoplus_{p \in \pi^{-1}(t)} \mathscr{C}_{t, p}, \quad \mathscr{C}:=\bigcup_{t \in T} \mathscr{C}_{t} .
$$

We want to show that $\mathscr{C}$ has the natural structure of a holomorphic vector bundle of $\operatorname{rank} \operatorname{ind}_{V, 0}(X)$ over $T$.

By Lemma 4.4, part ii, we may assume that the coordinates are chosen as in Lemma 4.4, part iii. Via $\pi$ we view $\mathscr{O}_{Z, 0} /(D F)$ as a finitely generated $\mathscr{O}_{T, 0}$-module and claim that

$$
\operatorname{depth}_{\mathscr{O}_{T, 0}} \frac{\mathscr{O}_{Z, 0}}{(D F)}=n-1 .
$$

For $k=1, \ldots, n-1$ let

$$
t_{k} g=0 \quad \text { in } \frac{\mathscr{O}_{Z, 0}}{\left(D F, t_{1}, \ldots, t_{k-1}\right)} .
$$

This means that there are representatives with

$$
t_{k} g=\alpha X_{t, 1} \quad \text { in } \frac{\mathscr{O}_{\mathbb{C}^{2 n-1}, 0}}{\left(f_{t, 1}, \ldots, f_{t, n-1}, t_{1}, \ldots, t_{k-1}, D F\right)} .
$$

Applying Cramer's rule to the tangency equation $X f=C f$ we obtain

$$
t_{k} g m_{2}=0 \quad \text { in } \frac{\mathscr{O}_{\mathbb{C}^{2 n-1}, 0}}{\left(f_{t, 1}, \ldots, f_{t, n-1}, t_{1}, \ldots, t_{k-1}, D F\right)} .
$$

By Lemma 4.4, part $\mathrm{i}$ and the choice of coordinates, $t_{k}$ and $m_{2}$ are not zero divisors in the last algebra since $\left(f_{t, 1}, \ldots, f_{t, n-1}, t_{1}, \ldots, t_{n-2}, D F, m_{2}\right)$ is a regular $\mathscr{O}_{\mathbb{C}^{2 n-1}, 0^{-}}$sequence. This shows the claim.

Now the Syzygy Theorem and the Auslander-Buchsbaum formula show that $\mathscr{O}_{Z, 0} /(D F)$ is a free $\mathscr{O}_{T, 0}$-module. We have an exact sequence of $\mathscr{O}_{T, 0}$-modules

$$
0 \rightarrow \frac{\mathscr{O}_{Z, 0}}{\operatorname{ann}_{\mathscr{O}_{Z, 0}}(D F)} \rightarrow \mathscr{O}_{Z, 0} \rightarrow \frac{\mathscr{O}_{Z, 0}}{(D F)} \rightarrow 0
$$

The Depth Lemma, see [JP00, 6.5.18], shows that

$$
\operatorname{depth}_{\mathscr{O}_{T, 0}} \frac{\mathscr{O}_{Z, 0}}{\operatorname{ann}_{\mathscr{O}_{Z, 0}}(D F)}=n-1,
$$

which by the Syzygy Theorem and the Auslander-Buchsbaum formula again means that the $\mathscr{O}_{T, 0}$-module $\mathscr{O}_{Z, 0} / \operatorname{ann}_{\mathscr{O}_{Z, 0}}(D F)$ is free. We have seen that the coherent $\mathscr{O}_{T}$-module

$$
\mathscr{F}:=\pi_{*} \mathscr{O}_{Z} / \operatorname{ann}_{\mathscr{O}_{Z}}(D F)
$$




\section{REAL AND COMPLEX INDICES OF VECTOR FIELDS ON CURVES}

is free for $T$ chosen small enough. By [Dou68] this is equivalent to the statement that the function $\nu: T \rightarrow \mathbb{N}$ defined by

$$
\nu(t):=\operatorname{dim}_{\mathbb{C}} \mathscr{F}_{t} \otimes_{\mathscr{O}_{T, t}} \mathbb{C}
$$

is constant. Now from the exact sequence

$$
0 \rightarrow \mathscr{F}_{t} \rightarrow\left(\pi_{*} \mathscr{O}_{Z}\right)_{t} \rightarrow\left(\pi_{*} \mathscr{O}_{Z} /(D F)\right)_{t} \rightarrow 0
$$

we obtain by tensoring with $\mathbb{C}$ the last part of the long exact sequence of torsion

$$
0 \rightarrow \mathscr{F}_{t} \otimes_{\mathscr{O}_{T, t}} \mathbb{C} \rightarrow \mathscr{B}_{t} \rightarrow \mathscr{B}_{t} /(D F) \rightarrow 0,
$$

where we have used that $\left(\pi_{*} \mathscr{O}_{Z} /(D F)\right)_{t}$ is a free $\mathscr{O}_{T, t}$-module, which is equivalent, see [Dou68], to the statement that

$$
\operatorname{Tor}_{1}^{\mathscr{O}_{T, t}}\left(\left(\pi_{*} \mathscr{O}_{Z} /(D F)\right)_{t}, \mathbb{C}\right)=0 .
$$

Since we also have an exact sequence

$$
0 \rightarrow \mathscr{C}_{t} \rightarrow \mathscr{B}_{t} \rightarrow \mathscr{B}_{t} /(D F) \rightarrow 0,
$$

this means that $\nu(t)=\operatorname{dim}_{\mathbb{C}} \mathscr{C}_{t}$ for all $t \in T$. By Lemma 3.3, for regular $t, \nu(t)$ equals the sum of Poincaré-Hopf indices of $X_{t}$ on the $t$-fibre of $f$ for $t \neq 0$ which is equal to ind ${ }_{V, 0}(X)$ and therefore $\operatorname{dim}_{\mathbb{C}} \mathscr{C}_{0}=\operatorname{ind}_{V, 0}(X)$. The map $\cdot D F: \mathscr{B} \rightarrow \mathscr{B}$ between vector bundles has constant rank and provides $\mathscr{C}$ with the natural structure of a holomorphic vector bundle.

\subsection{Examples and remarks}

We want to give a few examples of good vector fields on curves. We can always take the exterior product of the rows of the Jacobian matrix of $f$. In this way one obtains a vector field with isolated zero on the singularity, where the matrix $C$ is trivial and the index equals zero.

An example of a family of non-trivial good vector fields on a plane curve is the following:

$$
D_{k}: f=x^{2} y+y^{k-1}, \quad k \geqslant 4
$$

and

$$
X=(k-2) x^{m+1} \frac{\partial}{\partial x}+2 x^{m} y \frac{\partial}{\partial y}, \quad m \geqslant 3,
$$

with $c=2(k-1) x^{m}$. We have an exact sequence

$$
0 \rightarrow \operatorname{ann}_{\mathscr{B}_{0}}(D F) \rightarrow \mathscr{B}_{0} \stackrel{\cdot D F}{\longrightarrow} \mathscr{B}_{0} \rightarrow \frac{\mathscr{B}_{0}}{(D F)} \rightarrow 0
$$

and therefore

$$
\operatorname{dim}_{\mathbb{C}} \operatorname{ann}_{\mathscr{B}_{0}}(D F)=\operatorname{dim}_{\mathbb{C}} \frac{\mathscr{B}_{0}}{(D F)} .
$$

It is easy to compute that $\operatorname{dim}_{\mathbb{C}} \mathscr{B}_{0} /(D F)=2(k-1)$ and $\operatorname{dim}_{\mathbb{C}} \mathscr{B}_{0}=(k-1)(m+1)$ holds and therefore $\operatorname{ind}_{V, 0}(X)=(k-1)(m-1)$.

An example of a family of good vector fields on a space curve is

$$
f_{1}:=x^{2}+y^{2}+z^{2}, \quad f_{2}:=x y,
$$

with

Here we have

$$
X:=z^{l}(x-y)\left(x \frac{\partial}{\partial x}+y \frac{\partial}{\partial y}+z \frac{\partial}{\partial z}\right), \quad l \geqslant 1 .
$$

$$
C=\left(\begin{array}{cc}
2 z^{l}(x-y) & 0 \\
0 & 2 z^{l}(x-y)
\end{array}\right)
$$




\section{O. KLEHN}

In this case $\left(f_{1}, f_{2}, X_{3}\right)$ is a regular sequence and we have to permute the coordinates. This means that the index is given by the dimension of the factor space obtained from factoring the algebra $\mathscr{O}_{\mathbb{C}^{3}, 0} /\left(f_{1}, f_{2}, X_{3}\right)$ by the annihilator of

$$
\operatorname{det}\left(\begin{array}{cc}
2 x & 2 y \\
y & x
\end{array}\right)
$$

In this case we get a non-trivial index, which one may compute with computer algebra programs such as Singular [GPS01]. Note that $\operatorname{dim}_{\mathbb{C}} \mathscr{C}_{0}$ is always equal to $\operatorname{dim}_{\mathbb{C}} \mathscr{B}_{0}-\operatorname{dim}_{\mathbb{C}} \mathscr{B}_{0} /(D F)$. The last two dimensions are easy to compute with computer algebra.

We now want to explain why Theorem 1.4 is false in the general case of a complete intersection. Consider the case $n=3$ and $q=1$ and set $f_{i}:=\partial f / \partial z_{i}$ for $i=1,2,3$. We use the notation as in the proof of Theorem 1.4, i.e. $\mathscr{O}_{Z, 0}:=\mathscr{O}_{4,0} /\left(f-t, X_{t, 1}, X_{t, 2}\right)$ and $\left(f, X_{1}, X_{2}\right)$ is a regular sequence in $\mathscr{O}_{3,0}$, and consider the coherent $\mathscr{O}_{T}$-module $\pi_{*} \mathscr{O}_{Z} /\left(f_{3}\right)$ where $T$ is a small neighbourhood of 0 in $\mathbb{C}$. After shrinking $T$ we can assume that this sheaf is locally free over all $t \neq 0$. Obviously $t$ is not a zero divisor in $\mathscr{F}_{0}$ and therefore we have a law of conservation of numbers as in the proof of Theorem 1.4 and we get

$$
\operatorname{ind}_{V, 0}(X)=\operatorname{dim}_{\mathbb{C}} \mathscr{F}_{0} \otimes_{\mathscr{O}_{T, 0}} \mathbb{C} .
$$

We further have the exact sequence

$$
0 \rightarrow \operatorname{Tor}_{1}^{\mathscr{O}_{T, 0}}\left(\pi_{*} \mathscr{O}_{Z, 0} /\left(f_{3}\right), \mathbb{C}\right) \rightarrow \mathscr{F}_{0} \otimes_{\mathscr{O}_{T, 0}} \mathbb{C} \rightarrow \mathscr{B}_{0} \rightarrow \mathscr{B}_{0} /\left(f_{3}\right) \rightarrow 0
$$

and this means that

$$
\operatorname{ind}_{V, 0}(X)=\operatorname{dim}_{\mathbb{C}} \mathscr{C}_{0}+\operatorname{dim}_{\mathbb{C}} \operatorname{Tor}_{1}^{\mathscr{O}_{T, 0}}\left(\pi_{*} \mathscr{O}_{Z, 0} /\left(f_{3}\right), \mathbb{C}\right) .
$$

We now show that $\operatorname{dim}_{\mathbb{C}} \operatorname{Tor}_{1}^{\mathscr{O}_{T, 0}}\left(\pi_{*} \mathscr{O}_{Z, 0} /\left(f_{3}\right), \mathbb{C}\right)>0$ if the hypersurface is not smooth, which is equivalent to the statement that $t$ is a zero divisor in $\mathscr{O}_{Z, 0} /\left(f_{3}\right)$. Set $\mathscr{O}_{4,0}:=\mathbb{C}\left\{z_{1}, z_{2}, z_{3}, t\right\}$. The functions $\left(f-t, f_{1}, f_{2}, f_{3}\right)$ define an isolated zero in $\left(\mathbb{C}^{4}, 0\right)$ and therefore these define a regular $\mathscr{O}_{4,0}$-sequence. We have

$$
f_{1} X_{t, 1}+f_{2} X_{t, 2}=0
$$

in the ring $\mathscr{O}_{4,0} /\left(f-t, f_{3}\right)$. It follows that there are $\gamma_{1}, \gamma_{2} \in \mathscr{O}_{4,0}$ such that $X_{t, 1}=\gamma_{1} f_{2}$ and $X_{t, 2}=\gamma_{2} f_{1}$ in $\mathscr{O}_{4,0} /\left(f-t, f_{3}\right)$ holds. Inserting this in the tangency equation shows that there is a $\gamma \in \mathscr{O}_{4,0}$ such that $X_{t, 1}=\gamma f_{2}$ and $X_{t, 2}=-\gamma f_{1}$ in $\mathscr{O}_{4,0} /\left(f-t, f_{3}\right)$ holds. On the other hand $\mathscr{O}_{Z, 0} /\left(f_{3}, t\right)$ is artinian and therefore $\left(f-t, \gamma, f_{3}, t\right)$ is a weak regular $\mathscr{O}_{4,0}$-sequence. This means that we have an exact sequence

$$
0 \rightarrow \frac{\mathscr{O}_{4,0}}{\left(f-t, f_{1}, f_{2}, f_{3}\right)} \stackrel{\cdot \gamma}{\rightarrow} \frac{\mathscr{O}_{4,0}}{\left(f-t, \gamma f_{1}, \gamma f_{2}, f_{3}\right)} \rightarrow \frac{\mathscr{O}_{4,0}}{\left(f-t, \gamma, f_{3}\right)} \rightarrow 0
$$

with

$$
\frac{\mathscr{O}_{4,0}}{\left(f-t, \gamma f_{1}, \gamma f_{2}, f_{3}\right)} \cong \frac{\mathscr{O}_{Z, 0}}{\left(f_{3}\right)}
$$

The sequence shows that $\operatorname{depth}_{\mathscr{O}_{T, 0}} \mathscr{O}_{Z, 0} /\left(f_{3}\right)=0$. If $\gamma(0) \neq 0$ this is obvious. If $\gamma(0)=0$ we can apply the Depth Lemma using

$$
\operatorname{depth}_{\mathscr{O}_{T, 0}} \frac{\mathscr{O}_{4,0}}{\left(f-t, f_{1}, f_{2}, f_{3}\right)}=0
$$

and

$$
\operatorname{depth}_{\mathscr{O}_{T, 0}} \frac{\mathscr{O}_{4,0}}{\left(f-t, \gamma, f_{3}\right)}=1
$$




\section{REAL AND COMPLEX INDICES OF VECTOR FIELDS ON CURVES}

\section{A signature formula for the real index}

Now let

$$
\left(V^{\mathbb{R}}, 0\right):=\left(\left\{f_{1}^{\mathbb{R}}=\cdots=f_{q}^{\mathbb{R}}=0\right\}, 0\right) \subset\left(\mathbb{R}^{n}, 0\right)
$$

be a geometric complete intersection of dimension $n-q$, denote by $V$ and $f$ the complexifications and assume that $f$ defines an ICIS. Furthermore let the real analytic vector field $X^{\mathbb{R}}$ be tangent to $\left(V_{\mathbb{R}}, 0\right)$ with an algebraic isolated zero on $\left(V^{\mathbb{R}}, 0\right)$. As before $T$ is a small neighbourhood of the origin in $\mathbb{C}^{q}$ and let $T^{\mathbb{R}}$ be the corresponding subset in $\mathbb{R}^{q}$. We also assume that $X^{\mathbb{R}}$ is good in the real analytic sense. We keep the notation of the previous section for all complexifications and for real $t$ we denote the real algebra corresponding to $\mathscr{C}_{t, p}$ by $\mathscr{C}_{t, p}^{\mathbb{R}}$, if $\left.X^{\mathbb{R}}\right|_{V_{t}^{\mathbb{R}}}(p)=0$ and $p \in \mathbb{R}^{n}$ holds.

\subsection{Proof of Theorem 1.5}

First we prove a law of conservation of numbers for the signature. Theorem 1.5 then follows as a corollary. We also remark that the sufficient criterion for good vector fields also holds in the real analytic case. The proof is word for word as in the complex case.

Proposition 5.1. Let $q=n-1, X^{\mathbb{R}}$ be a good vector field and $l: \mathscr{C}_{0}^{\mathbb{R}} \rightarrow \mathbb{R}$ a linear form with $l\left(c_{1}\right)>0$, and for any regular value $t \in T^{\mathbb{R}}$ of $f^{\mathbb{R}}$ and any $p$ with $\left.X_{t}^{\mathbb{R}}\right|_{V_{t}^{\mathbb{R}}}(p)=0$ let $l_{t, p}: \mathscr{C}_{t, p}^{\mathbb{R}} \rightarrow \mathbb{R}$ be a linear form with $l_{t, p}\left(c_{t, p, 1}\right)>0$. Then

$$
\text { signature }\langle,\rangle_{l}=\sum_{\left\{\left.X_{t}^{\mathbb{R}}\right|_{V_{t}^{\mathbb{R}}}(p)=0\right\}} \text { signature }\langle,\rangle_{t, p},
$$

where the sum goes over the zeros tending to zero.

Proof. We consider the vector bundle $\mathscr{C}$ over $T$ defined in the proof of Theorem 1.4 and denote by $\tau$ the map given by complex conjugation. For any $t \in T^{\mathbb{R}}$ we consider the set of invariant multigerms $h \in \mathscr{C}_{t}$. These are the multigerms with $\tau \circ h=h \circ \tau$. We denote this set by $\mathscr{C}_{t}^{\mathbb{R}}$. We have

$$
\mathscr{C}_{t}^{\mathbb{R}}=\left(\oplus_{k} D_{k}\right) \oplus\left(\oplus_{l} E_{l}\right),
$$

where each component $D_{k}$ corresponds to an algebra $\mathscr{C}_{t, p_{k}}^{\mathbb{R}}$ for a real zero $p_{k}$ of $\left.X\right|_{V}$ and where each component

$$
E_{l}=\left(\mathscr{C}_{t, q_{l}} \oplus \mathscr{C}_{t, \overline{q_{l}}}\right)^{\mathbb{R}}
$$

corresponds to a pair of complex conjugated zeros and $\left(\mathscr{C}_{t, q_{l}} \oplus \mathscr{C}_{t, \overline{q_{l}}}\right)^{\mathbb{R}}$ is the subset of invariant elements of $\left(\mathscr{C}_{t, q_{l}} \oplus \mathscr{C}_{t, \overline{q_{l}}}\right)$. It consists of elements of the form

$$
h=\sum a_{I} z^{I}+\sum \overline{a_{I}} z^{I} .
$$

Here $q_{l}$ (respectively $\overline{q_{l}}$ ) are not real of course. If $\mu$ is the real dimension of $\mathscr{C}_{t}^{\mathbb{R}}$ then $\mu$ is given by $\operatorname{dim}_{\mathbb{C}} \mathscr{C}_{0}$. The set $\mathscr{C}^{\mathbb{R}}:=\bigcup_{t \in T^{\mathbb{R}}} \mathscr{C}_{t}^{\mathbb{R}}$ has, for $T$ chosen small enough, the natural structure of a real analytic vector bundle of rank $\mu$ over $T^{\mathbb{R}}$. We can continue $l$ real analytically to a family $l_{t}$ and obtain a real analytic family of non-degenerate bilinear forms $\langle,\rangle_{t_{t}}$. Equation (3) gives an orthogonal decomposition. By dividing the algebra $E_{l}$ by its maximal ideal one obtains $\mathbb{C}$ and therefore $E_{l}$ contributes nothing to the signature; see [EL77]. Therefore the signature of $\langle,\rangle_{l_{t}}$ is the sum of signatures of $\langle,\rangle_{t_{t}, p}$ that are defined as the restrictions to the components $D_{k}$. On the other hand we have $l_{t, p}\left(c_{t, p, 1}\right)>0$ and therefore the claim follows by continuity of signatures and by the Eisenbud-Levine Theorem if we choose a fixed regular value $t \in T^{\mathbb{R}}$ of $f$.

Proof of Theorem 1.5. For a good vector field the index counts the sum of indices of a good deformation of the vector field on a regular fibre in a neighbourhood of the origin by the properties 


\section{O. KLEHN}

of the real index given in Theorem 2.10 in [ASV98]. Now the claim follows from Lemma 3.5 and Proposition 5.1.

We want to consider an example. Let $f^{\mathbb{R}}(x, y):=x^{2}-y^{2}$ and $X^{\mathbb{R}}:=x^{2} \partial / \partial x+x y \partial / \partial y$. A good deformation is given by

$$
X_{t}^{\mathbb{R}}:=\left(x^{2}-t\right) \frac{\partial}{\partial x}+x y \frac{\partial}{\partial y}
$$

with $c_{t}=c=2 x$. Set $F_{t}:=V_{t}^{\mathbb{R}} \cap \overline{B_{\delta}}$ where $B_{\delta}$ is a small ball around the origin in $\mathbb{R}$. Then $F_{t}$ consists of two branches of a hyperbola and we have $\chi\left(F_{t}\right)=2$. If $l$ is a linear form as in Theorem 1.5 we obtain signature $\langle,\rangle_{l}=0$. Let $t=1, B_{\delta}:=\left\{x^{2}+y^{2}=3\right\}$ and $F:=F_{1}$. Then $X^{\mathbb{R}}$ deforms to

$$
\tilde{X}^{\mathbb{R}}:=\left(x^{2}-1\right) \frac{\partial}{\partial x}+x y \frac{\partial}{\partial y} .
$$

The boundary points of $F$ are $P_{1}=(\sqrt{2}, 1), P_{2}=(\sqrt{2},-1), P_{3}=(-\sqrt{2},-1)$ and $P_{4}=(-\sqrt{2}, 1)$. At the points $P_{1}$ and $P_{2}$ the vector field $\tilde{X}^{\mathbb{R}}$ points outwards, but inwards at the points $P_{3}$ and $P_{4}$. From the symmetry of the problem (only the directions of $\tilde{X}^{\mathbb{R}}$ are not symmetric) we find that the sum of the indices of $\tilde{X}^{\mathbb{R}}$ vanishes on $F$ and this is what Theorem 1.5 says. This can also be computed explicitly: the zeros of $\tilde{X}^{\mathbb{R}}$ on $F$ are $(-1,0)$ and $(1,0)$. We can parametrize both branches via $\varphi_{ \pm}(s):=\left( \pm \sqrt{1+s^{2}}, s\right)$ and write $\tilde{X}^{\mathbb{R}}$ in the coordinate given by $s$. One immediately sees that the index in $(-1,0)$ has the value -1 , and the value 1 in $(1,0)$.

If we want to count the Euler characteristic of $F_{t}$ we have to choose a good vector field whose deformation points outwards at all boundary points. This means that we have to choose a good vector field which points outwards at all boundary points of the intersection of the singular fibre with a small closed ball around the origin.

\subsection{Relations to results of Gómez-Mont and Mardesić}

Gómez-Mont and Mardesić have proven similar signature formulas [GM99, GM97]. These formulas hold for vector fields on isolated hypersurface singularities which have an isolated zero not only on the variety but also in the ambient space. We want to compare these formulas with our formula for vector fields on plane curves. Let $X$ be a real analytic vector field in $\left(\mathbb{R}^{n}, 0\right)$ with an isolated zero and $(V, 0):(\{f=0\}, 0) \subset\left(\mathbb{R}^{n}, 0\right)$ an odd-dimensional hypersurface with algebraic isolated singularity. Further let $X$ be tangent to $V$, i.e. $X f=c f$. We omit the upper $\mathbb{R}$ to indicate that we are working in the real analytic category. Define

$$
\mathbb{A}:=\frac{\mathscr{E}_{\mathbb{R}^{n}, 0}}{\left(f_{1}, \ldots f_{n}\right)} \quad \text { and } \quad \mathbb{B}:=\frac{\mathscr{E}_{\mathbb{R}^{n}, 0}}{\left(X_{1}, \ldots, X_{n}\right)}
$$

Here the $f_{i}$ are the partials of $f$. Let $H_{f}$ be the Hessian determinant of $f$. Then $\operatorname{det} D X$ and $H_{f}$ generate the socles of $\mathbb{B}$ (respectively $\mathbb{A}$ ). Now we have well determined classes

$$
H_{f}^{\mathrm{rel}}:=\frac{H_{f}}{c} \in \frac{\mathbb{A}}{\operatorname{ann}_{\mathbb{A}}(c)}, \quad \operatorname{det} D X^{\mathrm{rel}}:=\frac{\operatorname{det} D X}{c} \in \frac{\mathbb{B}}{\operatorname{ann}_{\mathbb{B}}(c)}
$$

defined in the obvious way, which generate the socles of these algebras. Let

$$
l_{1}: \frac{\mathbb{A}}{\operatorname{ann}_{\mathbb{A}}(c)} \rightarrow \mathbb{R}, \quad l_{2}: \frac{\mathbb{B}}{\operatorname{ann}_{\mathbb{B}}(c)} \rightarrow \mathbb{R}
$$

be linear forms with $l_{1}\left(H_{f}^{\text {rel }}\right)>0$ and $l_{2}\left(\operatorname{det} D X^{\text {rel }}\right)>0$. We have the following result.

Theorem 5.2 (Gómez-Mont and Mardesić).

$$
\operatorname{ind}_{V, 0}(X)=\text { signature }\langle,\rangle_{l_{2}}-\text { signature }\langle,\rangle_{l_{1}} \text {. }
$$




\section{REAL AND COMPLEX INDICES OF VECTOR FIELDS ON CURVES}

To compare this result with our theorem we additionally assume $n=2$, the $\mathscr{E}_{2,0}:=\mathbb{R}\{x, y\}$ sequence $\left(f, X_{1}\right)$ to be regular and the vector field to be good. We first give an explicit construction of all vector fields fulfilling both conditions. Denote the good deformation by $X_{t}$. We also set $\mathscr{E}_{3,0}:=\mathbb{R}\{x, y, t\}$ and $\mathscr{E}_{V, 0}:=\mathscr{E}_{3,0} /(f-t)$. The tangency condition gives

$$
X_{t, 1} f_{1}+X_{t, 2} f_{2}=0 \text { in } \mathscr{E}_{V, 0} .
$$

Since $\left(f_{1}, f_{2}\right)$ is a regular $\mathscr{E}_{V, 0}$-sequence, it follows immediately that there are $\tilde{\gamma}, \tilde{\delta_{1}}, \tilde{\delta}_{2} \in \mathscr{E}_{3,0}$ such that

$$
X_{t}=\left(\tilde{\gamma} f_{2}+\tilde{\delta_{1}}(f-t)\right) \frac{\partial}{\partial x}+\left(-\tilde{\gamma} f_{1}+\tilde{\delta_{2}}(f-t)\right) \frac{\partial}{\partial y} .
$$

Setting $t=0$ we obtain that there are $\delta_{1}, \delta_{2}, \gamma \in \mathscr{E}_{2,0}$ such that

$$
X=\left(\gamma f_{2}+\delta_{1} f\right) \frac{\partial}{\partial x}+\left(-\gamma f_{1}+\delta_{2} f\right) \frac{\partial}{\partial y} .
$$

We have $c=\delta_{1} f_{1}+\delta_{2} f_{2}$ and this means signature $\langle,\rangle_{l_{1}}=0$. We now claim that $\operatorname{ann}_{\mathbb{B}}(c)=\mathbb{B}(\gamma, f)$. Using

$$
\begin{aligned}
& c f=f_{1} X_{1}+f_{2} X_{2}, \\
& c \gamma=\delta_{2} X_{1}-\delta_{1} X_{2},
\end{aligned}
$$

we obtain $\mathbb{B}(\gamma, f) \subset \operatorname{ann}_{\mathbb{B}}(c)$. Now let $c g=\alpha_{1} X_{1}+\alpha_{2} X_{2}$. Multiplication with $f$ gives

$$
\left(f_{1} g-\alpha_{1} f\right) X_{1}+\left(f_{2} g-\alpha_{2} f\right) X_{2}=0 .
$$

Since $\left(X_{1}, X_{2}\right)$ is a regular $\mathscr{E}_{2,0}$-sequence there must be an $h \in \mathscr{E}_{2,0}$ with $f_{2} g-\alpha_{2} f=h X_{1}$. Since $\left(f, X_{1}\right)$ is a regular $\mathscr{E}_{2,0}$-sequence this also holds for $f, f_{2}$. Therefore we have $g=h \gamma$ in $\mathscr{E}_{2,0} /(f)$, which shows the claim. That $\mathscr{B}_{0}^{\mathbb{R}}(\gamma)=\operatorname{ann}_{\mathscr{B}_{0}^{\mathbb{R}}}\left(f_{2}\right)$ is obvious. We have obtained

$$
\mathscr{C}_{0}^{\mathbb{R}}=\frac{\mathbb{B}}{\operatorname{ann}_{\mathbb{B}}(c)}=\frac{\mathscr{E}_{2,0}}{(\gamma, f)} .
$$

To prove that Theorems 1.5 and 5.2 produce the same values one has to check that there is a positive real number $r$ such that $r c c_{1}=\operatorname{det} D X$ in $\mathbb{B}$. We have

$$
c \cdot c_{1}=c \text { trace } D X-c^{2}=c \operatorname{det} \frac{\partial(\gamma, f)}{\partial(x, y)}
$$

in $\mathbb{B}$. We verify the existence of such an $r$ in the following example.

Set $f:=x^{2}+y^{k+1}, \gamma:=y, \delta_{1}:=-(k+1), \delta_{2}:=y^{l}$. We have

$$
X_{1}=-(k+1) x^{2} \quad \text { and } \quad X_{2}=-2 x y+x^{2} y^{l}+y^{l+k+1} .
$$

One computes

$$
\operatorname{det} D X=-2(k+1)(l+k+1) x y^{l+k}
$$

and

in $\mathbb{B}$. Here we have $r=l+k+1$.

$$
c \operatorname{det} \frac{\partial(\gamma, f)}{\partial(x, y)}=-2(k+1) x y^{l+k}
$$

\section{ACKNOWLEDGEMENTS}

The author would like to thank W. Ebeling and X. Gómez-Mont for useful discussions about indices of vector fields. He is also grateful to D. van Straten for examples which showed that there was a mistake in the former proof of Theorem 1.4. Finally he also wants to thank the referees for useful and interesting comments on the paper. 


\section{REAL AND COMPLEX INDICES OF VECTOR FIELDS ON CURVES}

\section{REFERENCES}

ASV98 M. A. Aguilar, J. A. Seade and A. Verjovsky, Indices of vector fields and topological invariants of real analytic singularities, J. reine angew. Math. 504 (1998), 159-176.

AGV85 V. I. Arnold, S. M. Gusein-Zade and A. N. Varchenko, Singularities of differentiable maps I (Birkhäuser, Basel, 1985).

BG94 Ch. Bonatti and X. Gómez-Mont, The index of a holomorphic vector field on a singular variety I, Astérisque 222 (1994), 9-35.

Dou68 A. Douady, Flatness and privilege, Enseign. Math. (2) 14 (1968), 47-74.

EG99 W. Ebeling and S. M. Gusein-Zade, On the index of a vector field at an isolated singularity, in The Arnoldfest (Toronto, ON, 1997), Fields Inst. Commun., vol. 24 (Amer. Math. Soc., Providence, RI, 1999), 141-152.

EL77 D. Eisenbud and H. I. Levine, An algebraic formula for the degree of a $C^{\infty}$ map germ, Ann. of Math. (2) 106 (1977), 19-44.

Gom98 X. Gómez-Mont, An algebraic formula for the index of a vector field on a hypersurface with an isolated singularity, J. Algebraic Geom. 7 (1998), 731-752.

GM99 X. Gómez-Mont and P. Mardesić, Index of a vector field tangent to an odd-dimensional hypersurface and the signature of the relative Hessian, Funct. Anal. Appl. 33 (1999), 1-10.

GM97 X. Gómez-Mont and P. Mardesić, The index of a vector field tangent to a hypersurface and the signature of the relative Jacobian determinant, Ann. Inst. Fourier (Grenoble) 47 (1997), 1523-1539.

GSV91 X. Gómez-Mont, J. Seade and A. Verjovsky, The index of a holomorphic flow with an isolated singularity, Math. Ann. 291 (1991), 737-751.

GPS01 G. M. Greuel, G. Pfister and H. Schönemann, Singular 2.0. A computer algebra system for polynomial computations (Centre for Computer Algebra, University of Kaiserslautern, 2001).

GH78 Ph. Griffiths and J. Harris, Principles of algebraic geometry (Wiley, New York, 1978).

JP00 Th. de Jong and G. Pfister, Local analytic geometry (Vieweg, Braunschweig/Wiesbaden, 2000).

Kle02 O. Klehn, Local residues of holomorphic 1-forms on an isolated surface singularity, Manuscripta Math. 109 (2002), 93-108.

Kle03 O. Klehn, On the index of a vector field tangent to a hypersurface with non-isolated zero in the embedding space, Math. Nachr. 260 (2003), 48-57.

Loo84 E. J. N. Looijenga, Isolated singular points on complete intersections, London Mathematical Society Lecture Note Series, vol. 77 (Cambridge University Press, Cambridge, 1984).

LSS95 D. Lehmann, M. Soares and T. Suwa, On the index of a holomorphic vector field tangent to a singular variety, Bol. Soc. Brasil. Mat. 26 (1995), 183-199.

Oliver Klehn klehn@math.uni-hannover.de

Institut für Mathematik, Universität Hannover, Postfach 6009, D-30060 Hannover, Germany 